\begin{tabular}{|c|l|}
\hline Title & A coustic phonon modes and dispersion relations of nanowire superlattices \\
\hline Author(s) & Mizuno, Seiji; Nishiguchi, Norihiko \\
\hline Citation & Journal of Physics: Condensed Matter, 21/19), 195303 \\
\hline https://doi.org/10.1088/0953 8984/21/19/195303 \\
\hline Issue Date & 2009 \\
\hline Doc URL & http://hdl.handle.net/2115/47119 \\
\hline Rights & Copyright @2009 IOP Publishing Ltd. \\
\hline Type & article (author version) \\
\hline File Information & mizunoRevised2.pdf \\
\hline
\end{tabular}

Instructions for use 


\title{
Acoustic phonon modes and dispersion relations of nanowire superlattices
}

\author{
Seiji MIZUNO and Norihiko NISHIGUCHI \\ Department of Applied Physics, Graduate School of Engineering, \\ Hokkaido University, Sapporo 060-8628, Japan
}

(Dated: March 24, 2009)

\begin{abstract}
We study theoretically acoustic phonon modes in nanowire superlattices (NWSLs) composed of cubic materials. We classify the acoustic phonon modes in rectangular and square cross-section NWSLs, based on group theory. For NWSLs consisting of GaAs and AlAs, we calculate numerically the dispersion relations of each phonon mode and corresponding displacement fields. We examine the effects of both the lateral confinement and superlattice modulation along the wire axis. The results suggest that peculiar electron-phonon interactions occur, because the vibrations of both the lateral and longitudinal confining potentials induce scattering potential in addition to the deformation and piezoelectric potentials.
\end{abstract}




\section{INTRODUCTION}

Recent advances in nano fabrication technologies enable the synthesis of one-dimensional nanowire superlattices (NWSLs) made of various combinations of dissimilar materials, e.g. GaAs/GaP, Si/SiGe, InAs/InP and ZnSe/CdSe[1-6]. The hetero-structure modifies electron states in wire structure. The electronic states of the NWSLs were calculated with the oneband effective-mass theory.[7] This result shows that the NWSLs offer unique features, which are radically different from plain nanowires and quantum wells in their electronic, optical, and transport properties. A variety of possible optical and electrical applications utilizing the characteristics were also proposed[8-11].

In contrast, one pays little attention to modifications in the normal modes of phonons in the NWSLs. The phonons influence the electronic states and the transport properties via the electron-phonon interaction. Thus, the determination of the normal modes of phonons and spectra peculiar to the system are indispensable in order to understand the optical and electrical properties of the NWSLs and to predict the functions of devices using the NWSLs. However, the normal modes in the NWSLs are not well understood. A recent study of phonons in the GaN/AlN NWSL using Raman spectroscopy[11] shows an inexplicable peak in the phonon spectra, demanding further study on the phonon modes peculiar to the structure.

The fabricated NWSLs have various shapes of wire cross-section, e.g. triangular, rectangular, and hexagonal, manifesting elastic anisotropy of the constituent materials. Generally speaking, it is hard to derive analytically the phonon modes in the NWSLs. The only exception is acoustic torsional phonon modes in a cylindrical NWSL of elastically isotropic materials. One of the present authors investigated the dispersion relation and displacement field of the torsional modes, and examined the transmission of phonons in the NWSL using a potential theory and transfer matrix method[12, 13]. Although the work revealed the important aspects of phonon modes in the NWSL, they are limited to investigate the effects of phonons on the electronic system.

One of the purposes of this work is to give a method to derive acoustic phonon modes in a free-standing NWSL of anisotropic material with an arbitrary shape of cross-section. Then, we actually investigate the phonon modes in the rectangular and square cross-section NWSLs consisting of "anisotropic" materials, using the method. Based on group theory, 
we classify the acoustic phonon modes in these NWSLs since the classification is a powerful tool to discuss the electron-phonon interaction in further study of electron transport. For each mode, we calculate their phonon dispersion relations and displacement fields.

The outline of the present paper is as follows. In Sec. II, the eigenvalue equation giving the dispersion relations and displacement fields is presented. In Sec. III, we consider acoustic phonon modes in a rectangular cross-section NWSL consisting of cubic materials. Expressions for matrix elements in the eigenvalue equation are analytically calculated for this NWSL. Furthermore, the acoustic phonon modes are classified and discussed with the use of group theory. In Sec. IV, we consider a square cross-section NWSL consisting of cubic materials. In Sec. V, as a numerical example, we calculate the dispersion relations of rectangular and square cross-section NWSLs consisting of the alternate stacking of GaAs and AlAs. In Sec. VI, a summary and concluding remarks are given.

\section{GENERALIZED EIGENVALUE EQUATION}

In this section, we present the equation giving the eigenfrequencies of phonon modes in a freestanding NWSL. Acoustic phonon modes in the long wavelength limit obey the elastic wave equation,

$$
\rho \omega^{2} u_{i}+\sum_{j} \frac{\partial}{\partial x_{j}} \sigma_{i j}=0 .
$$

Here $u_{i}(i=x, y, z)$ is a displacement component, $\rho$ is the mass density, $\omega$ is frequency, and $\sigma_{i j}$ is the stress tensor given by

$$
\sigma_{i j}=\sum_{k, \ell} C_{i j k \ell} \frac{\partial}{\partial x_{k}} u_{\ell}
$$

where $C_{i j k \ell}$ is the stiffness tensor of the material. Inserting Eq. (2) into Eq. (1), we obtain

$$
\rho \omega^{2} u_{i}+\sum_{j, k, \ell} \frac{\partial}{\partial x_{j}}\left(C_{i j k \ell} \frac{\partial}{\partial x_{k}} u_{\ell}\right)=0 .
$$

We expand the displacement $u_{i}(\boldsymbol{r})$ in terms of a set of basis functions $\phi_{\alpha}(\boldsymbol{r})$,

$$
u_{i}(\boldsymbol{r})=\sum_{\alpha} A_{\alpha i} \phi_{\alpha}(\boldsymbol{r})
$$


Substituting Eq. (4) into Eq. (3), we have

$$
\rho \omega^{2} \sum_{\alpha} \phi_{\alpha}(\boldsymbol{r}) A_{\alpha i}+\sum_{\alpha, j, k, \ell} \frac{\partial}{\partial x_{j}}\left(C_{i j k \ell} \frac{\partial}{\partial x_{k}} \phi_{\alpha}(\boldsymbol{r})\right) A_{\alpha \ell}=0 .
$$

Multiplying Eq. (5) by $\phi_{\beta}^{*}(\boldsymbol{r})$ and integrating over the volume $V$, we obtain the generalized eigenvalue equation,

$$
\sum_{\alpha, \ell} H_{\beta i, \alpha \ell} A_{\alpha \ell}=\omega^{2} \sum_{\alpha, \ell} S_{\beta i, \alpha \ell} A_{\alpha \ell} .
$$

Here, the matrix elements $H_{\beta i, \alpha \ell}$ and $S_{\beta i, \alpha \ell}$ are defined by

$$
\begin{aligned}
H_{\beta i, \alpha \ell} & =\sum_{j, k}\left\langle j \beta\left|C_{i j k \ell}\right| k \alpha\right\rangle, \\
S_{\beta i, \alpha \ell} & =\delta_{i \ell}\langle\beta|\rho| \alpha\rangle,
\end{aligned}
$$

where

$$
\begin{aligned}
\left\langle j \beta\left|C_{i j k \ell}\right| k \alpha\right\rangle & =\int_{V} \frac{\partial \phi_{\beta}^{*}(\boldsymbol{r})}{\partial x_{j}} C_{i j k \ell} \frac{\partial \phi_{\alpha}(\boldsymbol{r})}{\partial x_{k}} d \boldsymbol{r}, \\
\langle\beta|\rho| \alpha\rangle & =\int_{V} \phi_{\beta}^{*}(\boldsymbol{r}) \rho \phi_{\alpha}(\boldsymbol{r}) d \boldsymbol{r} .
\end{aligned}
$$

Solving Eq. (6) with Eqs. (7) to (10), we can obtain the eigenfrequencies of phonons and corresponding phonon displacements in the NWSL. If $\rho$ is independent of $\boldsymbol{r}$ and the basis functions are orthogonal, the matrix $S_{\beta i, \alpha \ell}$ becomes diagonal. In NWSLs, however, $\rho$ and $C_{i j k \ell}$ depend on $\boldsymbol{r}$. Furthermore, we adopt non-orthogonal basis functions in the present work, as described below.

Hereafter, we consider NWSLs consisting of cubic materials. If the coordinate axes $x, y, z$ are chosen to coincide with the cubic crystal axes, its stiffness tensor is expressed as

$$
C_{i j k \ell}=C_{11} \delta_{i j} \delta_{k \ell} \delta_{i k}+C_{12} \delta_{i j} \delta_{k \ell}\left(1-\delta_{i k}\right)+C_{44} \delta_{i k} \delta_{j \ell}\left(1-\delta_{i j}\right)+C_{44} \delta_{i \ell} \delta_{j k}\left(1-\delta_{i k}\right)
$$

In this case, $H_{\beta i, \alpha \ell}$ can be explicitly written as

$$
\begin{aligned}
H_{\beta i, \alpha \ell} & =\left\{\left\langle i \beta\left|C_{11}\right| i \alpha\right\rangle+\sum_{j \neq i}\left\langle j \beta\left|C_{44}\right| j \alpha\right\rangle\right\} \delta_{i \ell} \\
& +\left\{\left\langle i \beta\left|C_{12}\right| \ell \alpha\right\rangle+\left\langle\ell \beta\left|C_{44}\right| i \alpha\right\rangle\right\}\left(1-\delta_{i \ell}\right) .
\end{aligned}
$$




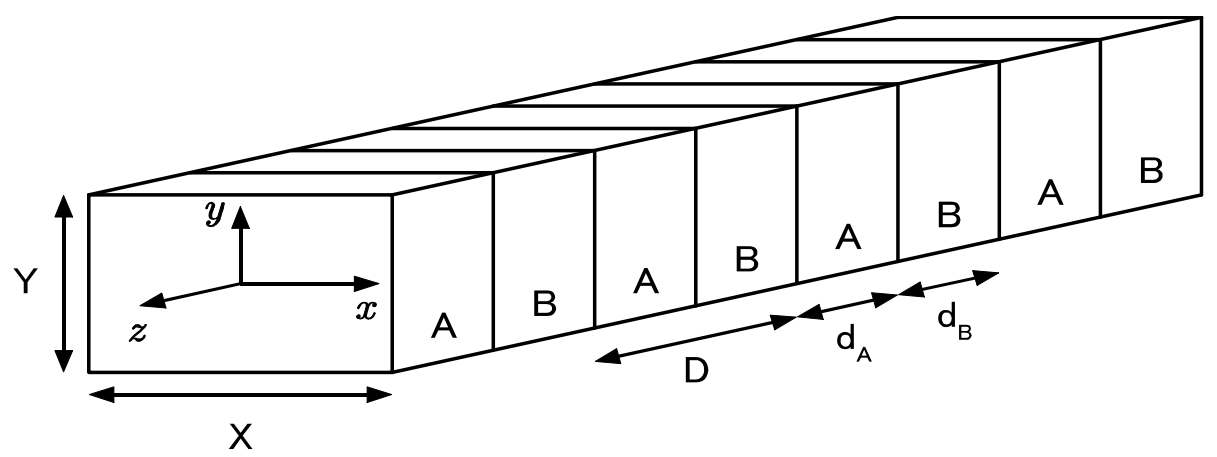

FIG. 1: Rectangular cross section NWSL

\section{RECTANGULAR CROSS-SECTION NWSL OF ANISOTROPIC MATERI-}

\section{ALS}

In this section, we consider acoustic phonon modes in a rectangular cross-section NWSL consisting of cubic materials [Fig. 1].

\section{A. Basis functions and matrix elements}

As basis functions, we choose the product of powers of the Cartesian coordinates in the $x y$ plane and the plane wave propagating along the $z$ axis:

$$
\phi_{\alpha}(q, \boldsymbol{r})=\frac{1}{\sqrt{V}}\left(\frac{2 x}{X}\right)^{m}\left(\frac{2 y}{Y}\right)^{n} e^{i(q+G) z}
$$

Here, $X$ and $Y$ denote the thicknesses of the wire in the $x$ and $y$ directions, respectively,

$$
V=S D=X Y D
$$

is the volume of the unit cell, where $S=X Y$ is the cross-section and $D$ is the length of the unit cell in the $z$ direction. Considering the periodicity in the $z$ direction, the $z$ dependence of the displacement component is expressed in the form of the Bloch wave in Eq. (13). $G$ is the reciprocal lattice vector determined by the periodicity $D$ of the NWSL,

$$
G=2 \pi k / D
$$

where $k$ is an integer. Thus, the basis functions are specified with $m, n$, and $G$ (or $k$ ), i.e., $\alpha=(m, n, G)$. 
The matrix elements $H_{\beta i, \alpha \ell}$ and $S_{\beta i, \alpha \ell}$, Eqs. (7) and (8), depend on the shape of the cross-section of the NWSL. Analytical expressions for these matrix elements can be easily calculated for the rectangular and square NWSLs. Another advantage of using Eq. (13) is that symmetry-adopted basis functions can be easily constructed.

Substituting Eq. (13) into Eq. (8), we have

$$
\begin{aligned}
S_{\beta i, \alpha \ell}(q) & =\delta_{i \ell} \frac{D}{V} \int\left(\frac{2 x}{X}\right)^{m+m^{\prime}} d x \int\left(\frac{2 y}{Y}\right)^{n+n^{\prime}} d y \frac{1}{D} \int_{0}^{D} \rho(z) e^{i\left(G-G^{\prime}\right) z} d z \\
& =\delta_{i \ell} F_{m+m^{\prime}, n+n^{\prime}} \rho\left(G-G^{\prime}\right) .
\end{aligned}
$$

Here, $\beta=\left(m^{\prime}, n^{\prime}, G^{\prime}\right), F_{m, n}$ is defined by

$$
\begin{aligned}
F_{m, n} & =\frac{1}{S} \int_{-X / 2}^{X / 2}\left(\frac{2 x}{X}\right)^{m} d x \int_{-Y / 2}^{Y / 2}\left(\frac{2 y}{Y}\right)^{n} d y \\
& =\frac{1}{(m+1)(n+1)} \delta_{m, \text { even }} \delta_{n, \text { even }}
\end{aligned}
$$

and $\rho(G)$ is the Fourier component of $\rho(z)$,

$$
\rho(G)=\frac{1}{D} \int_{0}^{D} \rho(z) e^{-i G z} d z .
$$

When $d_{A}=d_{B}=D / 2, \rho(G)$ is calculated as

$$
\rho(G)=\left\{\begin{array}{cl}
\frac{i}{\pi k}\left(\rho_{B}-\rho_{A}\right) \delta_{k, \text { odd }} & \text { for } G \neq 0 \\
\frac{\rho_{A}+\rho_{B}}{2} & \text { for } G=0
\end{array} .\right.
$$

where $\rho_{A}$ and $\rho_{B}$ are the mass densities of the constituent materials $\mathrm{A}$ and $\mathrm{B}$, respectively.

Substituting Eq. (13) into Eq. (12), we have

$$
\begin{aligned}
H_{\beta x, \alpha x}(q) & =C_{11}\left(G-G^{\prime}\right) \frac{4 m m^{\prime}}{X^{2}} F_{m^{\prime}+m-2, n^{\prime}+n}+C_{44}\left(G-G^{\prime}\right) \frac{4 n n^{\prime}}{Y^{2}} F_{m^{\prime}+m, n^{\prime}+n-2} \\
& +C_{44}\left(G-G^{\prime}\right)(q+G)\left(q+G^{\prime}\right) F_{m^{\prime}+m, n^{\prime}+n}, \\
H_{\beta y, \alpha y}(q) & =C_{44}\left(G-G^{\prime}\right) \frac{4 m m^{\prime}}{X^{2}} F_{m^{\prime}+m-2, n^{\prime}+n}+C_{11}\left(G-G^{\prime}\right) \frac{4 n n^{\prime}}{Y^{2}} F_{m^{\prime}+m, n^{\prime}+n-2} \\
& +C_{44}\left(G-G^{\prime}\right)(q+G)\left(q+G^{\prime}\right) F_{m^{\prime}+m, n^{\prime}+n}, \\
H_{\beta z, \alpha z}(q) & =C_{44}\left(G-G^{\prime}\right) \frac{4 m m^{\prime}}{X^{2}} F_{m^{\prime}+m-2, n^{\prime}+n}+C_{44}\left(G-G^{\prime}\right) \frac{4 n n^{\prime}}{Y^{2}} F_{m^{\prime}+m, n^{\prime}+n-2} \\
& +C_{11}\left(G-G^{\prime}\right)(q+G)\left(q+G^{\prime}\right) F_{m^{\prime}+m, n^{\prime}+n},
\end{aligned}
$$




$$
\begin{aligned}
& H_{\beta x, \alpha y}(q)=\left[C_{12}\left(G-G^{\prime}\right) m^{\prime} n+C_{44}\left(G-G^{\prime}\right) m n^{\prime}\right] \frac{4}{X Y} F_{m+m^{\prime}-1, n+n^{\prime}-1}, \\
& H_{\beta y, \alpha x}(q)=\left[C_{44}\left(G-G^{\prime}\right) m^{\prime} n+C_{12}\left(G-G^{\prime}\right) m n^{\prime}\right] \frac{4}{X Y} F_{m+m^{\prime}-1, n+n^{\prime}-1}, \\
& H_{\beta y, \alpha z}(q)=\left[C_{12}\left(G-G^{\prime}\right) n^{\prime}(q+G)-C_{44}\left(G-G^{\prime}\right) n\left(q+G^{\prime}\right)\right] \frac{2 i}{Y} F_{m+m^{\prime}, n+n^{\prime}-1}, \\
& H_{\beta z, \alpha y}(q)=\left[C_{44}\left(G-G^{\prime}\right) n^{\prime}(q+G)-C_{12}\left(G-G^{\prime}\right) n\left(q+G^{\prime}\right)\right] \frac{2 i}{Y} F_{m+m^{\prime}, n+n^{\prime}-1}, \\
& H_{\beta z, \alpha x}(q)=\left[C_{44}\left(G-G^{\prime}\right) m^{\prime}(q+G)-C_{12}\left(G-G^{\prime}\right) m\left(q+G^{\prime}\right)\right] \frac{2 i}{X} F_{m+m^{\prime}-1, n+n^{\prime}}, \\
& H_{\beta x, \alpha z}(q)=\left[C_{12}\left(G-G^{\prime}\right) m^{\prime}(q+G)-C_{44}\left(G-G^{\prime}\right) m\left(q+G^{\prime}\right)\right] \frac{2 i}{X} F_{m+m^{\prime}-1, n+n^{\prime}} .
\end{aligned}
$$

Here, $C_{i j}(G)$ is the Fourier component of $C_{i j}(z)$,

$$
C_{i j}(G)=\frac{1}{D} \int_{0}^{D} C_{i j}(z) e^{-i G z} d z
$$

When $d_{A}=d_{B}=D / 2, C_{i j}(G)$ is calculated as

$$
C_{i j}(G)=\left\{\begin{array}{ccc}
\frac{i}{\pi k}\left(C_{i j}^{B}-C_{i j}^{A}\right) \delta_{k, \text { odd }} & \text { for } & G \neq 0 \\
\frac{C_{i j}^{A}+C_{i j}^{B}}{2} & \text { for } \quad G=0
\end{array},\right.
$$

where $C_{i j}^{A}$ and $C_{i j}^{B}$ are the stiffness constants of the constituent materials A and $\mathrm{B}$, respectively.

In general, a large number of basis functions are necessary to express the phonon displacements. By considering the symmetry of the system, however, the number of basis functions, i.e., the size of the matrices $H_{\beta x, \alpha x}(q)$ and $S_{\beta x, \alpha x}(q)$, can be reduced, as described below.

\section{B. Symmetry and acoustic phonon modes}

In this section, we utilize group theory[14, 15] and classify the acoustic phonon modes in the rectangular NWSL. The translational symmetry along the wire axis has been already considered by introducing the wave numbers $q$ and $G$. While the point group of the unit cell is $D_{2 h}$, the group of $q$ is $C_{2 v}$ for $0<|q|<\pi / D$. The character table of $C_{2 v}$ is shown in Table I.[14]

Using projection operators obtained with the character table of $C_{2 v}$, we can construct symmetry-adopted basis functions belonging to the irreducible representations of this group. The projection operators corresponding to the irreducible representations are constructed 


\begin{tabular}{ccccc}
$C_{2 v}$ & $E$ & $C_{2}$ & $\sigma_{y}$ & $\sigma_{x}$ \\
\hline \hline $\mathrm{A}_{1}$ & 1 & 1 & 1 & 1 \\
\hline $\mathrm{A}_{2}$ & 1 & 1 & -1 & -1 \\
\hline $\mathrm{B}_{1}$ & 1 & -1 & 1 & -1 \\
\hline $\mathrm{B}_{2}$ & 1 & -1 & -1 & 1
\end{tabular}

TABLE I: Character table of $C_{2 v}$

as

$$
\begin{aligned}
& P_{A_{1}}=E+C_{2}+\sigma_{y}+\sigma_{x}, \\
& P_{A_{2}}=E+C_{2}-\sigma_{y}-\sigma_{x}, \\
& P_{B_{1}}=E-C_{2}+\sigma_{y}-\sigma_{x}, \\
& P_{B_{2}}=E-C_{2}-\sigma_{y}+\sigma_{x} .
\end{aligned}
$$

The obtained basis functions are as follows:

$$
\begin{aligned}
& \mathrm{A}_{1}: u_{x}=x ; u_{y}=y ; u_{z}=\left\{x^{2}, y^{2}\right\} \\
& \mathrm{A}_{2}: u_{x}=y ; u_{y}=x ; u_{z}=x y \\
& \mathrm{~B}_{1}: u_{x}=\left\{x^{2}, y^{2}\right\} ; u_{y}=x y ; u_{z}=x, \\
& \mathrm{~B}_{2}: u_{x}=x y ; u_{y}=\left\{x^{2}, y^{2}\right\} ; u_{z}=y .
\end{aligned}
$$

These modes are schematically illustrated in Fig. 2, in which the phonon displacements at the corners of the rectangular cross-section are shown. Figure 2 shows only the symmetry of the phonon displacements in the NWSL. Actual displacements are determined by solving the eigenvalue equation (6).

Considering the symmetry of the basis functions shown in Eqs. (35) to (38), we express the displacement components as

$$
\begin{aligned}
& u_{x}(q, \boldsymbol{r})=\frac{1}{\sqrt{V}} \sum_{\alpha} A_{\alpha, x}(q)\left(\frac{2 x}{X}\right)^{2 s+1}\left(\frac{2 y}{Y}\right)^{2 t} e^{i(q+G) z} \\
& u_{y}(q, \boldsymbol{r})=\frac{1}{\sqrt{V}} \sum_{\alpha} A_{\alpha, y}(q)\left(\frac{2 x}{X}\right)^{2 s}\left(\frac{2 y}{Y}\right)^{2 t+1} e^{i(q+G) z} \\
& u_{z}(q, \boldsymbol{r})=\frac{1}{\sqrt{V}} \sum_{\alpha} A_{\alpha, z}(q)\left(\frac{2 x}{X}\right)^{2 s}\left(\frac{2 y}{Y}\right)^{2 t} e^{i(q+G) z}
\end{aligned}
$$



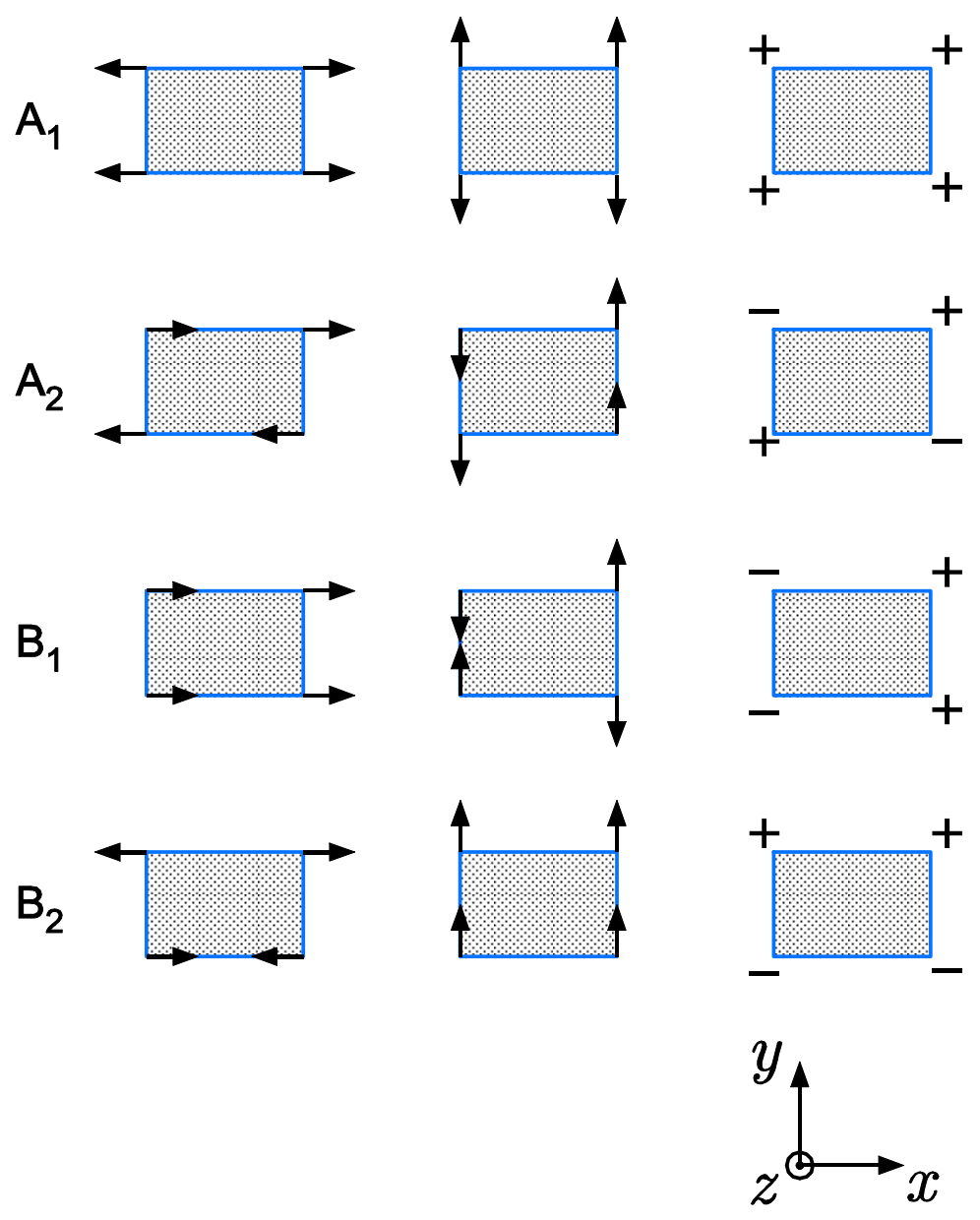

FIG. 2: Symmetry-adopted basis functions belonging to the irreducible representations of $\mathrm{C}_{2 v}$. Arrows and \pm show the phonon displacements at the corners in the $x y$ plane and in the $z$ direction, respectively.

for the $A_{1}$ mode,

$$
\begin{aligned}
& u_{x}(q, \boldsymbol{r})=\frac{1}{\sqrt{V}} \sum_{\alpha} A_{\alpha, x}(q)\left(\frac{2 x}{X}\right)^{2 s}\left(\frac{2 y}{Y}\right)^{2 t+1} e^{i(q+G) z} \\
& u_{y}(q, \boldsymbol{r})=\frac{1}{\sqrt{V}} \sum_{\alpha} A_{\alpha, y}(q)\left(\frac{2 x}{X}\right)^{2 s+1}\left(\frac{2 y}{Y}\right)^{2 t} e^{i(q+G) z} \\
& u_{z}(q, \boldsymbol{r})=\frac{1}{\sqrt{V}} \sum_{\alpha} A_{\alpha, z}(q)\left(\frac{2 x}{X}\right)^{2 s+1}\left(\frac{2 y}{Y}\right)^{2 t+1} e^{i(q+G) z}
\end{aligned}
$$


for $\mathrm{A}_{2}$ mode,

$$
\begin{aligned}
& u_{x}(q, \boldsymbol{r})=\frac{1}{\sqrt{V}} \sum_{\alpha} A_{\alpha, x}(q)\left(\frac{2 x}{X}\right)^{2 s}\left(\frac{2 y}{Y}\right)^{2 t} e^{i(q+G) z} \\
& u_{y}(q, \boldsymbol{r})=\frac{1}{\sqrt{V}} \sum_{\alpha} A_{\alpha, y}(q)\left(\frac{2 x}{X}\right)^{2 s+1}\left(\frac{2 y}{Y}\right)^{2 t+1} e^{i(q+G) z} \\
& u_{z}(q, \boldsymbol{r})=\frac{1}{\sqrt{V}} \sum_{\alpha} A_{\alpha, z}(q)\left(\frac{2 x}{X}\right)^{2 s+1}\left(\frac{2 y}{Y}\right)^{2 t} e^{i(q+G) z}
\end{aligned}
$$

for $\mathrm{B}_{1}$ mode, and

$$
\begin{aligned}
& u_{x}(q, \boldsymbol{r})=\frac{1}{\sqrt{V}} \sum_{\alpha} A_{\alpha, x}(q)\left(\frac{2 x}{X}\right)^{2 s+1}\left(\frac{2 y}{Y}\right)^{2 t+1} e^{i(q+G) z} \\
& u_{y}(q, \boldsymbol{r})=\frac{1}{\sqrt{V}} \sum_{\alpha} A_{\alpha, y}(q)\left(\frac{2 x}{X}\right)^{2 s}\left(\frac{2 y}{Y}\right)^{2 t} e^{i(q+G) z} \\
& u_{z}(q, \boldsymbol{r})=\frac{1}{\sqrt{V}} \sum_{\alpha} A_{\alpha, z}(q)\left(\frac{2 x}{X}\right)^{2 s}\left(\frac{2 y}{Y}\right)^{2 t+1} e^{i(q+G) z}
\end{aligned}
$$

for $\mathrm{B}_{2}$ mode, where $\alpha=(s, t, G)$.

These modes are decoupled from each other. This can be directly confirmed, by substituting Eqs. (39) to (50) into Eqs.(20) to (28).

\section{SQUARE NWSL OF ANISOTROPIC MATERIAL}

In this section, we consider acoustic phonon modes in a square cross-section NWSL. By setting $X=Y$ in the results obtained in Sec. III, we can calculate the phonon dispersion relations of this wire. However, symmetry-adopted basis functions are different from those of the rectangular wire. The group of $q$ becomes $C_{4 v}$ for the square cross-section NWSL. The character table of $C_{4 v}$ is shown in Table 2.[14]

The projection operators are constructed as

$$
\begin{aligned}
& P_{A_{1}}=E+C_{4}+C_{4}^{-1}+C_{4}^{2}+\sigma_{x}+\sigma_{y}+\sigma_{d}+\sigma_{d^{\prime}}, \\
& P_{A_{2}}=E+C_{4}+C_{4}^{-1}+C_{4}^{2}-\sigma_{x}-\sigma_{y}-\sigma_{d}-\sigma_{d^{\prime}}, \\
& P_{B_{1}}=E-C_{4}-C_{4}^{-1}+C_{4}^{2}+\sigma_{x}+\sigma_{y}-\sigma_{d}-\sigma_{d^{\prime}}, \\
& P_{B_{2}}=E-C_{4}-C_{4}^{-1}+C_{4}^{2}-\sigma_{x}-\sigma_{y}+\sigma_{d}+\sigma_{d^{\prime}}, \\
& P_{E}^{(1)}=E-C_{4}^{2}-\sigma_{x}+\sigma_{y}, \\
& P_{E}^{(2)}=E-C_{4}^{2}+\sigma_{x}-\sigma_{y} .
\end{aligned}
$$




\begin{tabular}{ccccccc}
$C_{4 v}$ & $E$ & $2 C_{4}$ & $C_{4}^{2}$ & $2 \sigma_{v}$ & $2 \sigma_{d}$ compatibility relations with $C_{2 v}$ \\
\hline \hline $\mathrm{A}_{1}$ & 1 & 1 & 1 & 1 & 1 & $\mathrm{~A}_{1}\left(C_{2 v}\right)$ \\
\hline $\mathrm{A}_{2}$ & 1 & 1 & 1 & -1 & -1 & $\mathrm{~A}_{2}\left(C_{2 v}\right)$ \\
\hline $\mathrm{B}_{1}$ & 1 & -1 & 1 & 1 & -1 & $\mathrm{~A}_{1}\left(C_{2 v}\right)$ \\
\hline $\mathrm{B}_{2}$ & 1 & -1 & 1 & -1 & 1 & $\mathrm{~A}_{2}\left(C_{2 v}\right)$ \\
\hline $\mathrm{E}$ & 2 & 0 & -2 & 0 & 0 & $\mathrm{~B}_{1}\left(C_{2 v}\right)+\mathrm{B}_{2}\left(C_{2 v}\right)$ \\
\hline
\end{tabular}

TABLE II: Character table of $C_{4 v}$

With the use of these operators, we obtain the basis functions belonging to the irreducible representations of $C_{4 v}$ :

$$
\begin{aligned}
& \mathrm{A}_{1}: u_{x}=x, u_{y}=y ; u_{z}=x^{2}+y^{2}, \\
& \mathrm{~A}_{2}: u_{x}=y, u_{y}=-x ; u_{z}=x y\left(x^{2}-y^{2}\right), \\
& \mathrm{B}_{1}: u_{x}=x, u_{y}=-y ; u_{z}=x^{2}-y^{2}, \\
& \mathrm{~B}_{2}: u_{x}=y, u_{y}=x ; u_{z}=x y\left(x^{2}+y^{2}\right), \\
& \mathrm{E}: u_{x}=\left\{x^{2}, y^{2}\right\} ; u_{y}=x y ; u_{z}=x \\
& \quad: u_{x}=x y ; u_{y}=\left\{x^{2}, y^{2}\right\} ; u_{z}=y .
\end{aligned}
$$

These modes are schematically illustrated in Fig. 3.

Considering the symmetry shown in Eqs. (57) to (62), we can construct the basis functions, as in the rectangular cross-section NWSL. Here, we note that both $\mathrm{A}_{1}$ and $\mathrm{B}_{1}$ modes in $\mathrm{C}_{4 v}$ correspond to $\mathrm{A}_{1}$ mode in $\mathrm{C}_{2 v}$, i.e., the forms (57) and (59) are included in Eqs. (39) to (41). Thus, it is convenient to use Eqs. (39) to (41) for both $A_{1}$ and $B_{1}$ modes in the actual numerical calculation. As a reference, the compatibility relations with $\mathrm{C}_{2 v}$ are shown in Table II. $\mathrm{B}_{1}$ modes can be distinguished from $\mathrm{A}_{1}$ mode by the calculated displacement components. Similarly, Eqs. (42) to (44) are available for $\mathrm{A}_{2}$ and $\mathrm{B}_{2}$ modes in the square cross-section NWSL. Also, Eqs. (45) to (47) and Eqs. (48) to (50) are used for E mode.

Here, we stress that $A_{1}$ and $B_{1}$ modes are symmetrically different modes in the square cross-section wire, though both $\mathrm{A}_{1}$ and $\mathrm{B}_{1}$ modes in a square cross-section "plain" nanowire are named dilatational modes in a paper[17], where these modes were classified based on the symmetry of the rectangular cross-section wire. 

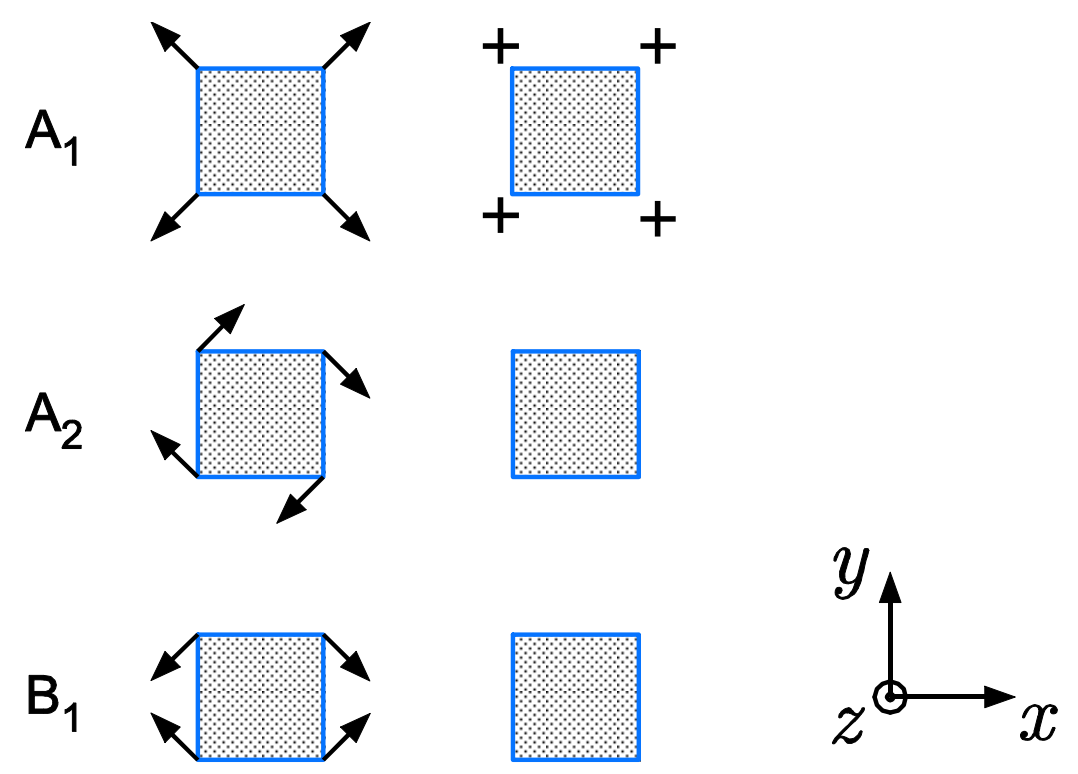

$\mathrm{B}_{2}$
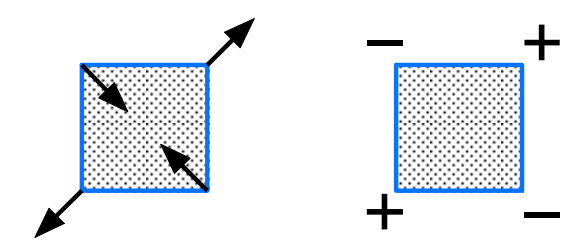

E
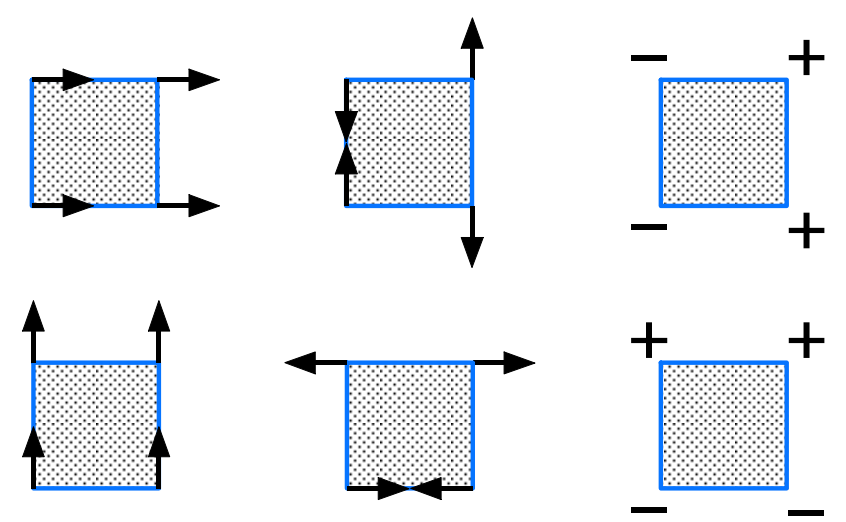

FIG. 3: Symmetry-adopted basis functions belonging to the irreducible representations of $\mathrm{C}_{4 v}$. Arrows and \pm show the phonon displacements at the corners in the $x y$ plane and in the $z$ direction, respectively. 


\section{NUMERICAL EXAMPLES}

\section{A. rectangular cross-section NWSL}

As the first numerical example, we consider a rectangular cross-section NWSL consisting of the alternate stacking of GaAs and AlAs $(A=$ GaAs and $B=A l A s)$. The size of the NWSL is assumed to be $X=100 \AA, Y=50 \AA$, and $d_{A}=d_{B}=D / 2=40 \AA$. Other parameters we used are as follows: $\rho=3.76 \mathrm{~g} / \mathrm{cm}^{3}, C_{11}=120.2, C_{12}=57.0$, and $C_{44}=58.9$ (all in units of $10^{10} \mathrm{dyn} / \mathrm{cm}^{2}$ ) for AlAs; $\rho=5.36 \mathrm{~g} / \mathrm{cm}^{3}, C_{11}=118.8, C_{12}=53.8$, and $C_{44}=59.6$ (all in units of $10^{10} \mathrm{dyn} / \mathrm{cm}^{2}$ ) for GaAs.[16]

The phonon dispersion relations calculated for four modes are shown in Fig. 4. In the present calculation, the number of basis functions at each $q$ point is empirically selected to be 336 for each mode. These dispersion relations reflect the effects of both the confinement of phonons in the lateral direction and superlattice modulation in the longitudinal direction.

The overall structure of each phonon dispersion relation in Fig. 4 can be approximately understood by the folding of the dispersion curves for a homogeneous cylinder into a miniBrillouin zone (BZ) determined by the periodicity $D$ of the NWSL.

Subband structure exists in the dispersion relation of the homogeneous plain nanowire. This is due to the fact that the wave vectors in the lateral direction are discretized because of the lateral confinement. For comparison, we show in Fig. 5 the dispersion relations calculated for the GaAs plain nanowire with the same cross-section as the present NWSL.

In the present example, the second mode at $q=0$ in each dispersion relation of the NWSL originates from the second subband of the plain nanowire. On the other hand, the third mode at $q=0$ originates from folding of the lowest subband of the plain nanowire.

Figure 4(a) shows the dispersion relations of $\mathrm{A}_{1}$ modes. The calculated displacement patterns corresponding to the lowest, second, and third modes at $q=0$ are illustrated in Fig. 6(a). In this figure, the arrows show the phonon displacements projected into the $x y$ plane. The lowest mode shows a dilatation (or contraction) in the diagonal directions, while the second and third modes show a dilatation in the $x$ direction. Displacements for higher frequency modes are more complicated patterns.

Figure 4(b) shows the dispersion relations of $\mathrm{A}_{2}$ modes. The displacement patterns corresponding to the lowest three modes at $q=0$ are illustrated in Fig. 6(b). This figure 

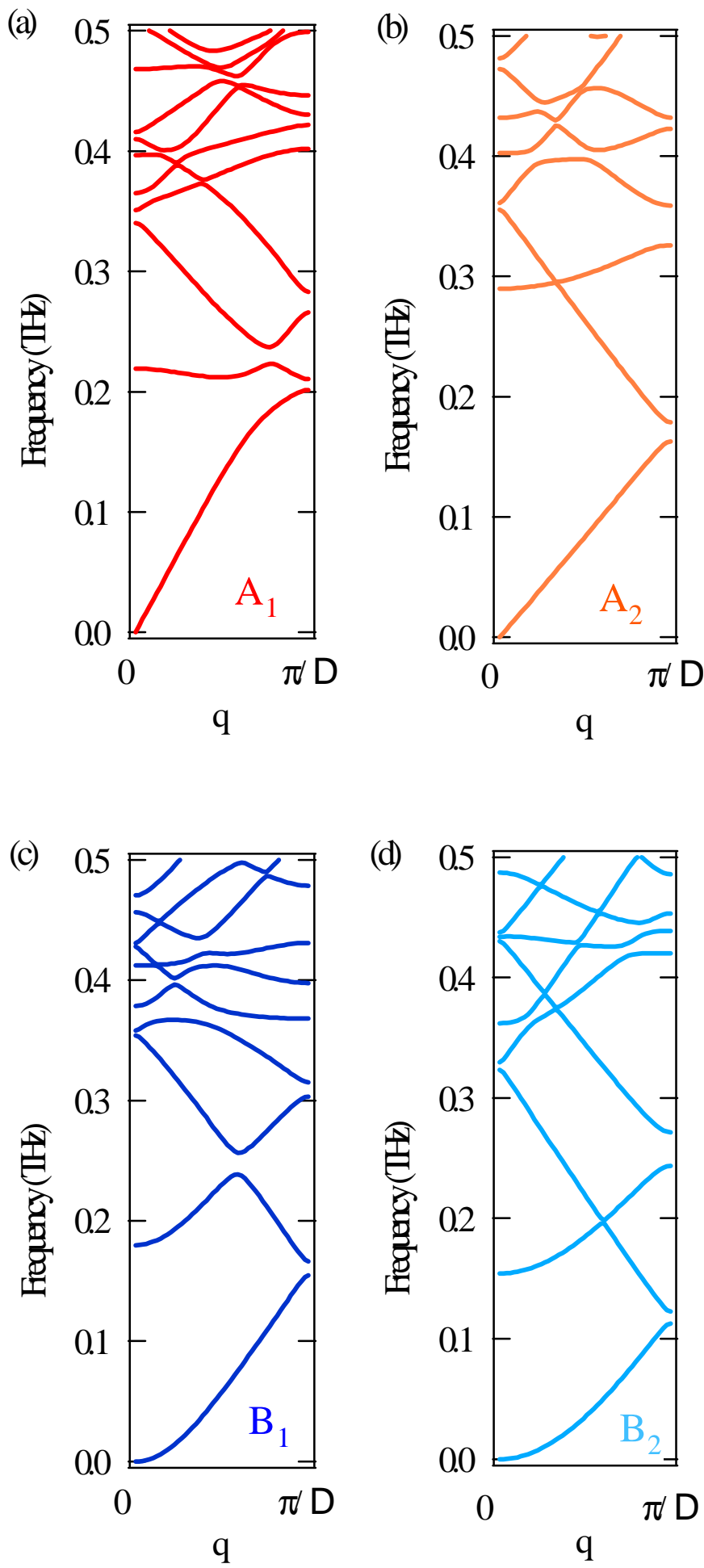

FIG. 4: Phonon dispersion relations of the rectangular cross-section NWSL consisting of GaAs and AlAs. The size of the NWSL is assumed to be $X=100 \AA, Y=50 \AA$, and $d_{A}=d_{B}=D / 2=40$ $\AA$. 

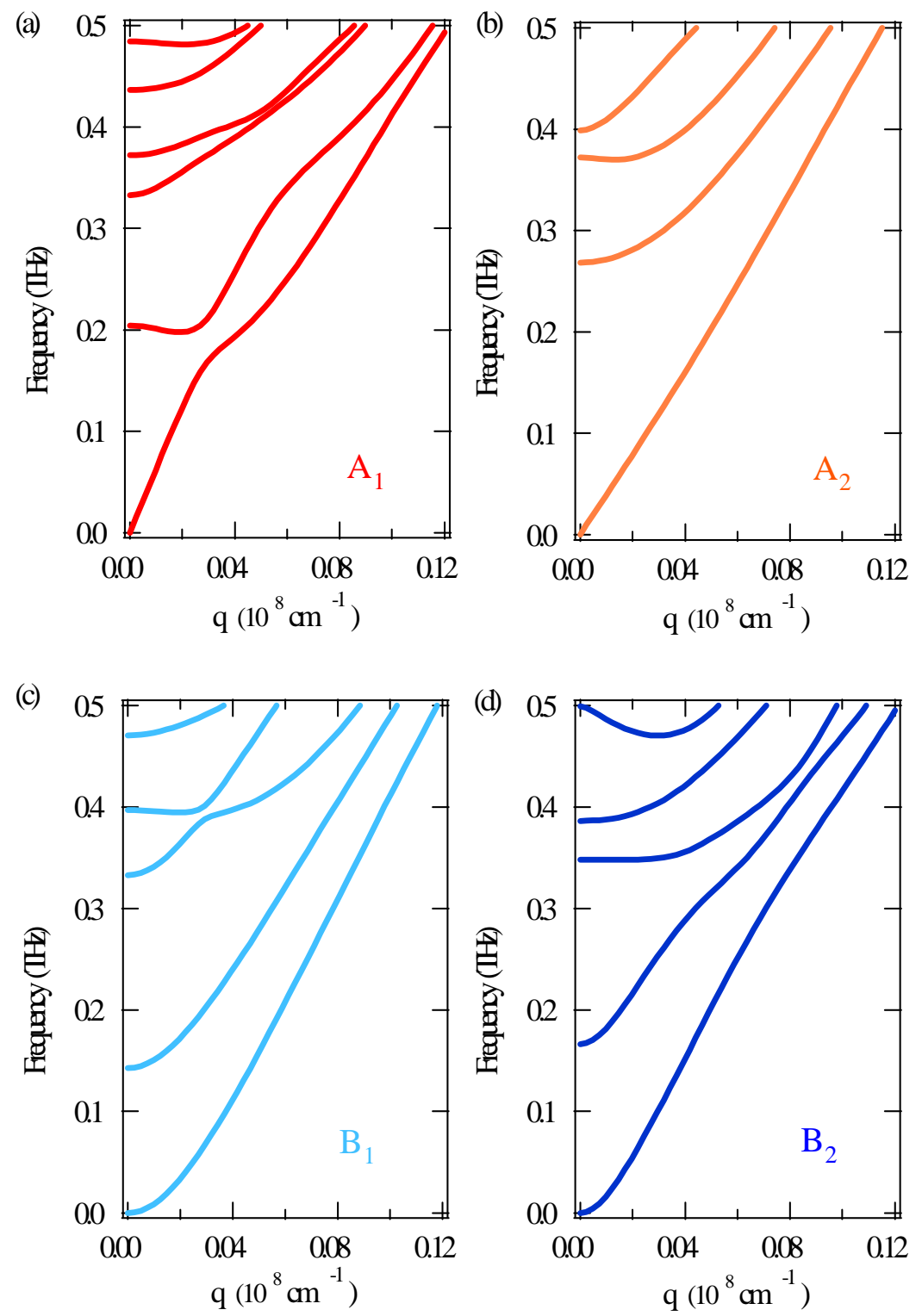

FIG. 5: Phonon dispersion relations of the rectangular cross-section GaAs plain nanowire. The size of the plain nanowire is assumed to be $X=100 \AA$ and $Y=50 \AA$.

shows the feature of a torsional mode. The lowest mode shows rotation about the wire axis. In the second mode, on the other hand, there are two centers of rotation.

Figures $4(\mathrm{c})$ and $4(\mathrm{~d})$ show the dispersion relations of $\mathrm{B}_{1}$ and $\mathrm{B}_{2}$ modes, respectively, and Figs. 6(c) and 6(d) illustrate their displacement patterns corresponding to the lowest three modes at $q=0$. The lowest modes have the feature of a flexural mode, which shows uniform displacement in the $x$ or $y$ direction. Displacements for higher frequency modes 
(a) A1 mode

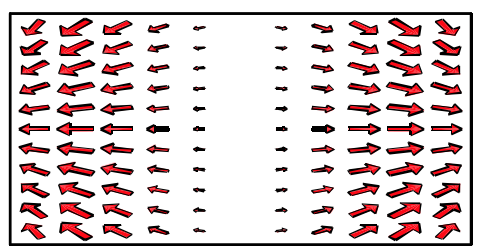

$\omega=0.34 \mathrm{THz}$

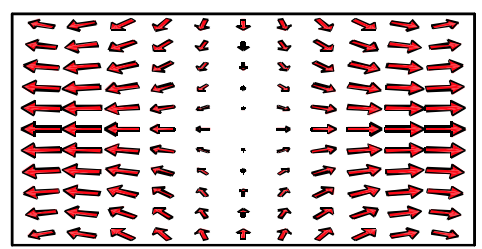

$\omega=0.22 \mathrm{THz}$

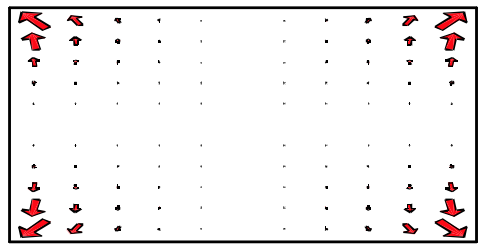

$\omega=0 \mathrm{THz}$

(c) B1 mode

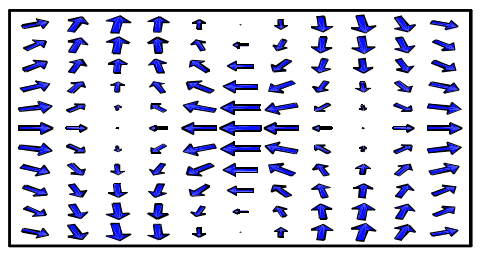

$\omega=0.35 \mathrm{THz}$

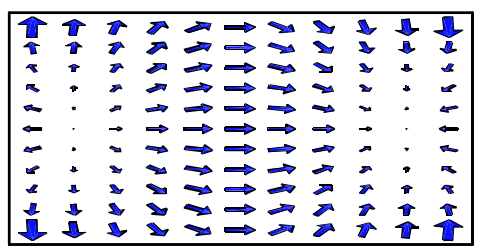

$\omega=0.17 \mathrm{THz}$

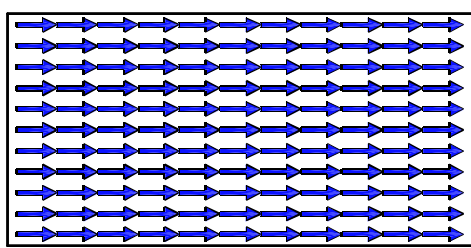

$\omega=0 \mathrm{THz}$ (b) A2 mode

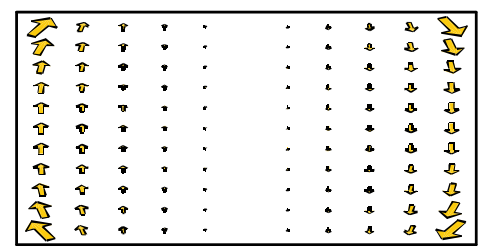

$\omega=0.35 \mathrm{THz}$

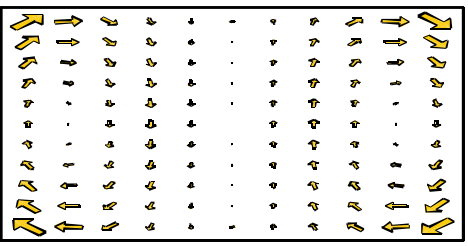

$\omega=0.29 \mathrm{THz}$

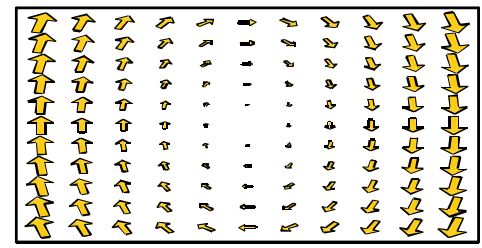

$\omega=0 \mathrm{THz}$

(d) B2 mode

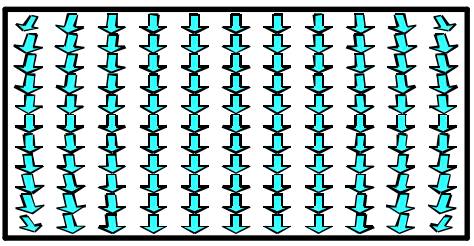

$\omega=0.32 \mathrm{THz}$

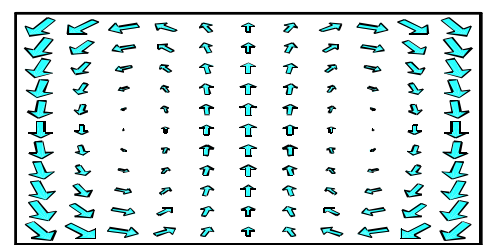

$\omega=0.15 \mathrm{THz}$

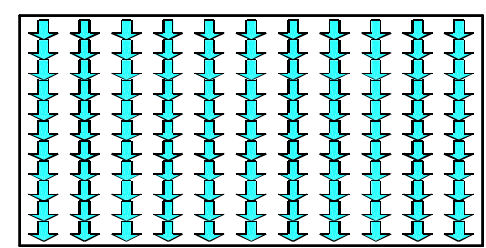

$\omega=0 \mathrm{THz}$

FIG. 6: Displacement patterns corresponding to the lowest and second dispersion curves at $q=0$. 
become more complicated patterns.

For small $q$, the lowest dispersion curves of $\mathrm{A}_{1}$ and $\mathrm{A}_{2}$ modes are linear in $q$. On the other hand, the lowest dispersion curves of $\mathrm{B}_{1}$ and $\mathrm{B}_{2}$ modes are proportional to $q^{2}$. These linear and parabolic behabiors are well known in the plain nanowire.[17]

\section{B. square cross-section NWSL}

As the second example, we consider a square cross-section NWSL consisting of GaAs and AlAs. The size of this NWSL is assumed to be $X=70 \AA, Y=70 \AA$, and $d_{A}=d_{B}=D / 2=$ $40 \AA$. The dispersion relations calculated for five modes are shown in Fig. 7.
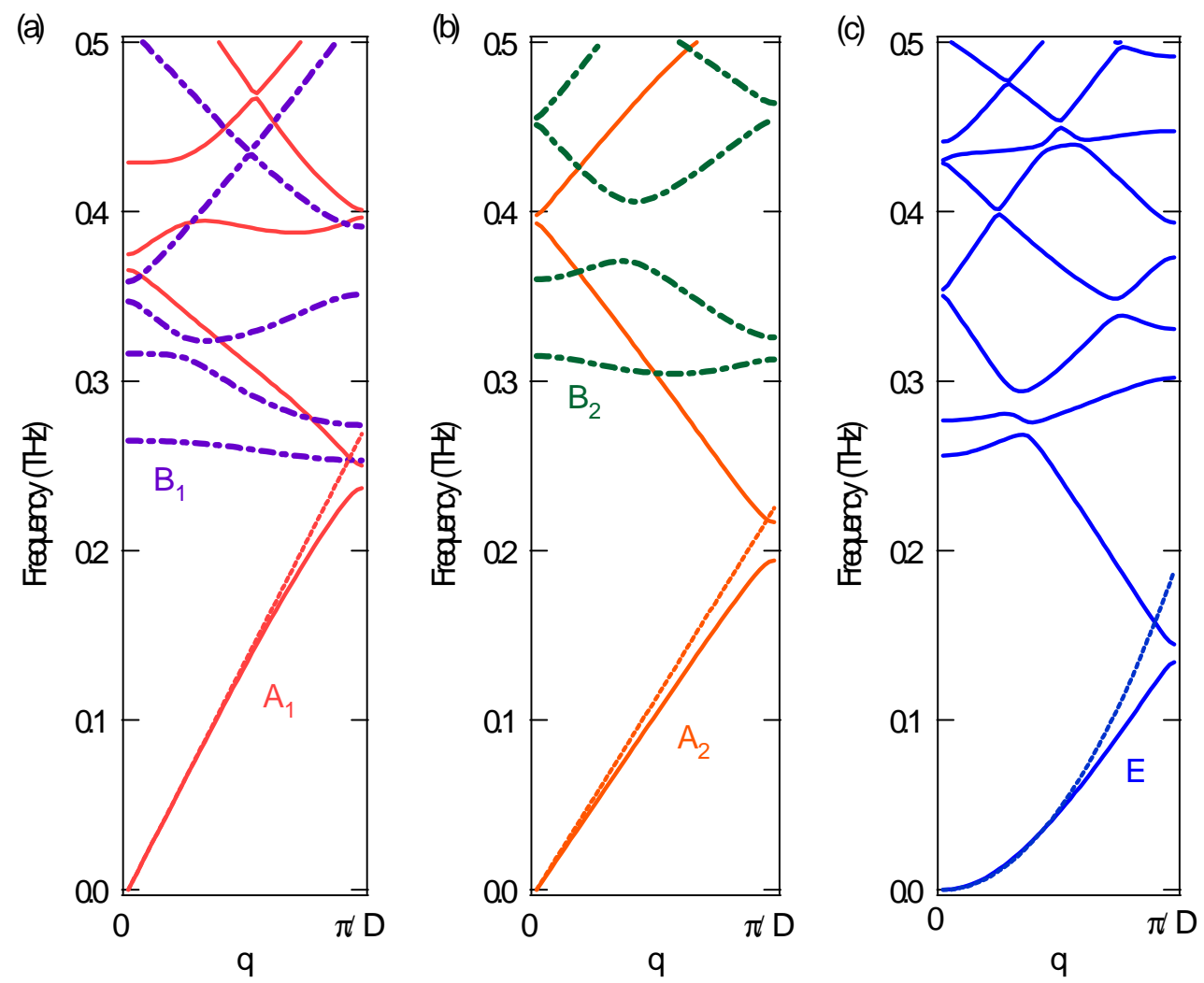

FIG. 7: Phonon dispersion relations of the square cross-section NWSL consisting of GaAs and AlAs: (a) $\mathrm{A}_{1}$ mode (dilatational mode, solid lines) and $\mathrm{B}_{1}$ mode (dashed lines); (b) $\mathrm{A}_{2}$ mode (torsional mode, solid lines) and $\mathrm{B}_{2}$ mode (shear mode, dashed lines); (c) E modes (flexural modes). The size of the NWSL is assumed to be $X=Y=70 \AA$, and $d_{A}=d_{B}=D / 2=40 \AA$. The dotted lines are calculated with the approximated formulas. In (c), all dispersion curves are doubly degenerated. 
Figure $7(\mathrm{a})$ shows the dispersion relations of $\mathrm{A}_{1}$ and $\mathrm{B}_{1}$ modes. The solid and dashed lines shows $A_{1}$ and $B_{1}$ modes, respectively. In the lowest dispersion curve of $A_{1}$ mode, $\omega$ vanishes at $q=0$. On the other hand, the lowest frequency of $\mathrm{B}_{1}$ mode has a finite value at $q=\pi / D$.

The calculated displacement patterns corresponding to the lowest two of $\mathrm{A}_{1}$ and $\mathrm{B}_{1}$ modes at $q=0$ are illustrated in Figs. 8(a) and 8(c), respectively. In these figures, the arrows show the phonon displacements projected into the $x y$ plane. These figures clearly show that the lowest $\mathrm{A}_{1}$ mode has the feature of a dilatational mode, i.e., a dilatation (or contraction) of the square cross-section in both the $x$ and $y$ directions, while the lowest $\mathrm{B}_{1}$ mode shows the alternating dilatation and contraction in the $x$ and $y$ directions.

Figure $7(\mathrm{~b})$ shows the dispersion relations of $\mathrm{A}_{2}$ and $\mathrm{B}_{2}$ modes. The displacement patterns corresponding to the lowest two of $\mathrm{A}_{2}$ and $\mathrm{B}_{2}$ modes at $q=0$ are illustrated in Fig. 8(b) and $8(\mathrm{~d})$, respectively. These figures clearly show the features of torsional and shear modes. The torsional mode shows rotation about the wire axis, and the shear mode alternating stretching in the two diagonal directions.

Figure 7(c) shows the dispersion relations of E modes. All dispersion curves are doubly degenerate because the irreducible representation of $\mathrm{E}$ mode is two-dimensional, as shown in Table II. The displacement patterns corresponding to the lowest and second modes at $q=0$ are illustrated in Fig. 8(e). These figures clearly show the features of flexural modes.

For E modes, the lowest dispersion curve near $q=0$ is proportional to $q^{2}$. This parabolic behavior is due to the fact that the flexural modes correspond to bending. The analytical form of the dispersion relation of bending mode in the plain wire is known to be $\omega \propto q^{2}$ in the long-wavelength limit.[18, 19] On the other hand, the lowest dispersion curves of $\mathrm{A}_{1}$ and $\mathrm{A}_{2}$ mode are linear in $q$ in the long wavelength limit.

In the Appendix, we derive the explicit expressions for the dispersion relations of $A_{1}$, $\mathrm{A}_{2}$, and $\mathrm{E}$ modes in the long wavelength limit. These expressions are compared with the numerical results in Fig. 7. The approximated result reproduces the numerical dispersion curve near the zone center. 
(a) A1 mode

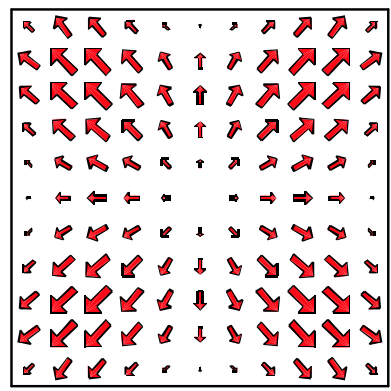

$\omega=0.36 \mathrm{THz}$

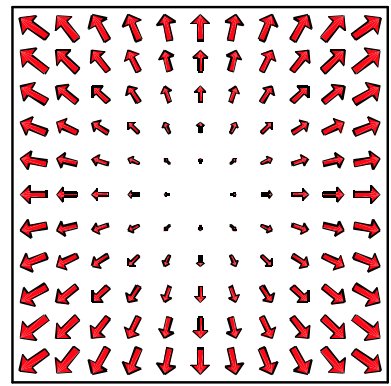

$\omega=0 \mathrm{THz}$

(d) B2 mode

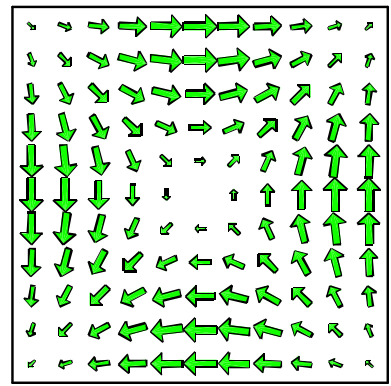

$\omega=0.36 \mathrm{THz}$

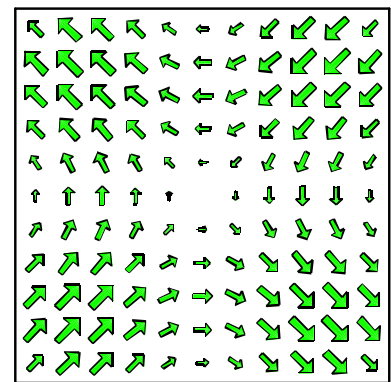

$\omega=0.31 \mathrm{THz}$ (b) A2 mode

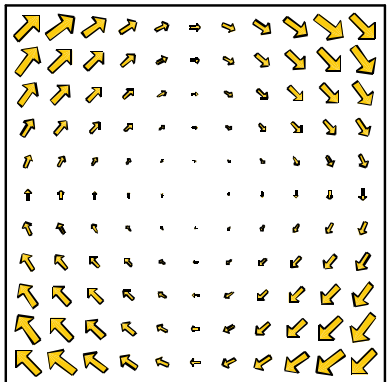

$\omega=0.39 \mathrm{THz}$

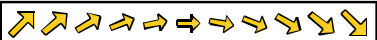

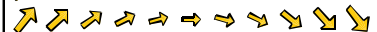
$\nabla \nabla \nabla \Delta \Rightarrow \Rightarrow \nabla \nabla \nabla \square$

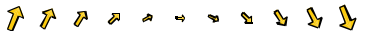

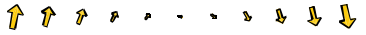
रिิ

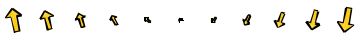

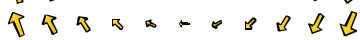

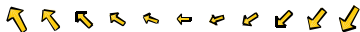

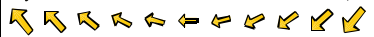

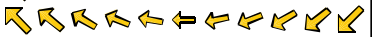

$\omega=0 \mathrm{THz}$

(e) E mode

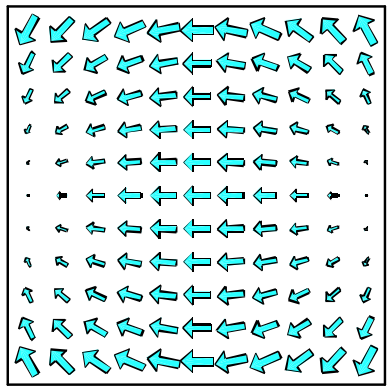

$\omega=0.25 \mathrm{THz}$

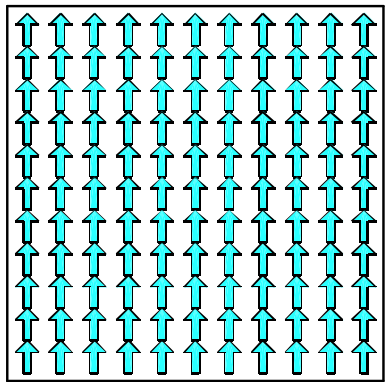

$\omega=0 \mathrm{THz}$ (c) B1 mode

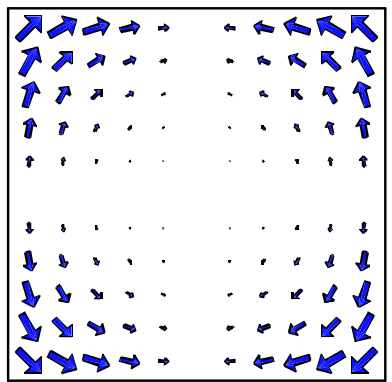

$\omega=0.31 \mathrm{THz}$

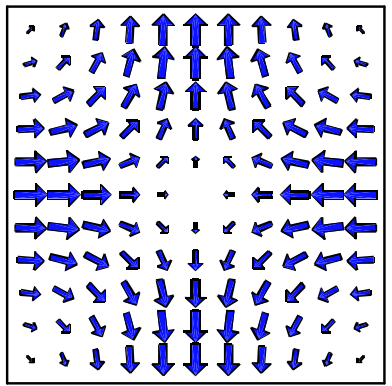

$\omega=0.26 \mathrm{THz}$

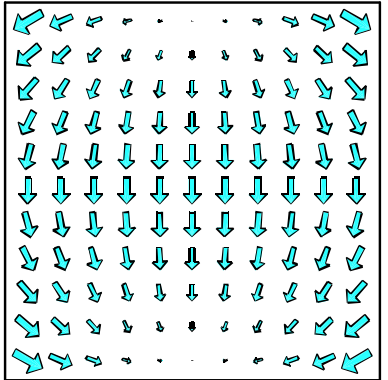

$\omega=0.25 \mathrm{THz}$

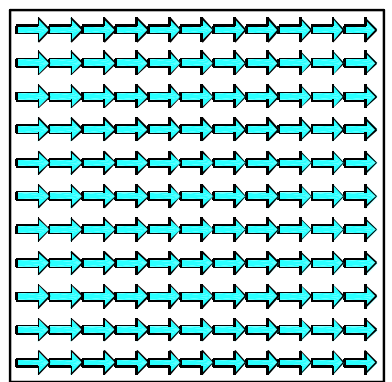

$\omega=0 \mathrm{THz}$

FIG. 8: Displacement pattern correspomding to the lowest and second dispersion curves at $q=0$. 


\section{SUMMARY AND CONCLUDING REMARKS}

In the present paper, we theoretically studied the acoustic phonon modes in NWSLs composed of cubic materials. Based on group theory, we classified the acoustic phonon modes in rectangular and square cross-section GaAs/AlAs NWSLs and calculated dispersion relations and displacement fields. The results provide fundamental understandings of the acoustic phonon modes in the NWSLs.

The acoustic phonon modes in the square cross-section NWSL are classified into five types, i.e., $\mathrm{A}_{1}$ (dilatational), $\mathrm{A}_{2}$ (torsional), $\mathrm{B}_{1}, \mathrm{~B}_{2}$ (shear), and $\mathrm{E}$ (flexural) modes. In the rectangular NWSL, the shear mode is mixed with the torsional mode since they are not distinguished owing to lower symmetry. Similarly, the dilatational mode is mixed with the $\mathrm{B}_{1}$ mode. In addition, the two-fold degeneracy of $\mathrm{E}$ mode is removed, i.e., the flexural mode in the $x$ direction is distinguished from that in the $y$ direction. The dilatational, torsional, shear, and flexural modes in plain nanowires with the same cross-section shapes were discussed in a previous paper[17]. Our result shows that there is another mode (i.e., $\mathrm{B}_{1}$ mode) in the square cross-section NWSL and also in the square plain nanowire.

The phonon dispersion relation of each mode reflects the effects of both the superlattice modulation and the lateral confinement of phonons. The overall structure of the phonon dispersion relations can be approximately understood by the folding of dispersion curves of a homogeneous plain wire, whose dispersion relations have subband structure because the wave vectors in the lateral direction are discretized due to the lateral confinement of phonons. The dispersion curves of the plain wire are folded into the mini-BZ determined by the periodicity along the wire axis, and the frequency gaps are generated.

All the phonon modes in the NWSLs contain both longitudinal and transverse wave components. The former is responsible for electron-phonon interactions via the deformation potential and the latter contributes to electron scatterings via piezoelectric coupling. In addition to these bulk electron-phonon interactions, the acoustic phonons are expected to induce non-local electron-phonon interactions peculiar to quasi-low-dimensional structure. That is, electron energy levels due to quantum confinement are modified by the acoustic phonons because the cross section of the system changes[20-22]. This type of interaction is referred to as the ripple mechanism[21] or the macroscopic deformation potential[22]. This interaction affects remarkably electron scattering in thin quantum wires in collaboration 
with the deformation potential[20]. The effects are also anticipated for electrons confined in the NWSLs, which will lead to electron energy dissipation and transport properties different from those in plain nanowires. These effects for the NWSLs will be discussed elsewhere.

\section{Acknowledgments}

The authors would like to thank S. Tamura, and Y. Nakamura for useful discussions. This work is supported in part by a grant-in-aid for scientific research from the Ministry of Education, Culture, Sports, Science and Technology of Japan (Grant No. 1965106507 and Grant No. 20246094).

\section{APPENDIX A: ANALYTICAL EXPRESSIONS FOR THE LOWEST DISPER- SION CURVES IN THE LONG WAVELENGTH LIMIT}

In this appendix, we derive the explicit expressions for the lowest dispersion curves of the dilatational, torsional and flexural modes in the square cross-section NWSL in the long wavelength limit.

For the dilatational mode, the basis function corresponding to the lowest dispersion curve of the long wavelength phonon can be approximated as

$$
\begin{aligned}
& u_{x}=x e^{i q z}, \\
& u_{y}=y e^{i q z}, \\
& u_{z}=e^{i q z},
\end{aligned}
$$

because the terms of the lowest power and $G=0$ are dominant in Eqs. (39) to (41). Calculating the matrix elements (Eqs. (8) and (12)) with Eqs. (A1) to (A3), we have a 
simple expression for the eigenvalue equation,

$$
\begin{aligned}
& \left(\begin{array}{ccc}
\frac{4}{X^{2}} C_{11}(0)+\frac{C_{44}(0)}{3} q^{2} & \frac{4}{X^{2}} C_{12}(0) & \frac{2 i}{X} C_{12}(0) q \\
\frac{4}{X^{2}} C_{12}(0) & \frac{4}{X^{2}} C_{11}(0)+\frac{C_{44}(0)}{3} q^{2} & \frac{2 i}{X} C_{12}(0) q \\
-\frac{2 i}{X} C_{12}(0) q & -\frac{2 i}{X} C_{12}(0) q & C_{11}(0) q^{2}
\end{array}\right)\left(\begin{array}{c}
A_{x} \\
A_{y} \\
A_{z}
\end{array}\right) \\
& =\left(\begin{array}{ccc}
\frac{1}{3} \rho(0) \omega^{2} & 0 & 0 \\
0 & \frac{1}{3} \rho(0) \omega^{2} & 0 \\
0 & 0 & \rho(0) \omega^{2}
\end{array}\right)\left(\begin{array}{c}
A_{x} \\
A_{y} \\
A_{z}
\end{array}\right) .
\end{aligned}
$$

Solving Eq. (A4), we have the dispersion relation for the long wavelength phonon as

$$
\omega=v_{d i l} q
$$

where

$$
v_{d i l}=\sqrt{\frac{Y}{\rho(0)}},
$$

and

$$
Y=\frac{C_{11}(0)^{2}+C_{11}(0) C_{12}(0)-2 C_{12}(0)^{2}}{C_{11}(0)+C_{12}(0)}
$$

Eq. (A7) is in agreement with Young's modulus determined by the average stiffness constants $C_{11}(0)$ and $C_{12}(0)$, which are given in Eq. (30).

For the torsional mode, the basis function corresponding to the lowest dispersion curve of the long wavelength phonon can be approximated as

$$
\begin{aligned}
& u_{x}=y e^{i q z} \\
& u_{y}=-x e^{i q z} \\
& u_{z}=0
\end{aligned}
$$

Calculating the matrix elements (Eqs. (8) and (12)) with Eqs. (A8) to (A10), we have a simple expression for the eigenvalue equation,

$$
\left(\begin{array}{cc}
C_{44}(0)\left(\frac{4}{X^{2}}+\frac{q^{2}}{3}\right) & C_{44}(0) \frac{4}{X^{2}} \\
C_{44}(0) \frac{4}{X^{2}} & C_{44}(0)\left(\frac{4}{X^{2}}+\frac{q^{2}}{3}\right)
\end{array}\right)\left(\begin{array}{c}
A_{x} \\
A_{y}
\end{array}\right)=\frac{\rho(0)}{3} \omega^{2}\left(\begin{array}{c}
A_{x} \\
A_{y}
\end{array}\right)
$$

Solving Eq. (A11), we have the dispersion relation near $q=0$ :

$$
\omega=v_{\text {tor }} q
$$


where

$$
v_{\text {tor }}=\sqrt{\frac{C_{44}(0)}{\rho(0)}} .
$$

Equation (A13) shows the torsional mode corresponds to the pure transverse mode whose velocity is determined by the average mass density $\rho(0)$ and average stiffness constant $C_{44}(0)$.

For the flexural mode, the basis function corresponding to the lowest dispersion curve around $q=0$ is approximated as

$$
\begin{aligned}
& u_{x}=e^{i q z} \\
& u_{y}=x y e^{i q z} \\
& u_{z}=x e^{i q z}
\end{aligned}
$$

In this case, the eigenvalue equation becomes

$$
\begin{aligned}
& \left(\begin{array}{ccc}
C_{44}(0) q^{2} & 0 & -\frac{2 i}{X} C_{44}(0) q \\
0 & \frac{4}{3 X^{2}}\left(C_{44}(0)+C_{11}(0)\right)+\frac{C_{44}(0)}{9} q^{2} & \frac{2 i}{3 X} C_{12}(0) q \\
\frac{2 i}{X} C_{44}(0) q & -\frac{2 i}{3 X} C_{12}(0) q & \frac{4}{X^{2}} C_{44}(0)+\frac{C_{11}(0)}{3} q^{2}
\end{array}\right)\left(\begin{array}{c}
A_{x} \\
A_{y} \\
A_{z}
\end{array}\right) \\
& =\left(\begin{array}{ccc}
\rho(0) \omega^{2} & 0 & 0 \\
0 & \frac{1}{9} \rho(0) \omega^{2} & 0 \\
0 & 0 & \frac{1}{3} \rho(0) \omega^{2}
\end{array}\right)\left(\begin{array}{c}
A_{x} \\
A_{y} \\
A_{z}
\end{array}\right) .
\end{aligned}
$$

Solving Eq. (A17), we obtain the explicit expression for the dispersion relation

$$
\begin{aligned}
\omega & =\sqrt{\frac{\alpha}{\rho(0)}} X q^{2}, \\
\alpha & =\frac{C_{44}(0)^{2}+C_{11}(0) C_{44}(0)-C_{12}(0)^{2}}{12\left(C_{12}(0)+C_{44}(0)\right)}
\end{aligned}
$$

If we use basis function of another form

$$
\begin{aligned}
& u_{x}=x y e^{i q z} \\
& u_{y}=e^{i q z} \\
& u_{z}=y e^{i q z}
\end{aligned}
$$

the same results as Eqs. (A18) and (A19) are obtained. This is due to the fact that the 
irreducible representation of $\mathrm{E}$ mode is two-dimensional.

[1] M. S. Gudikson L. J. Lauhon, J. Wang, D. C. Smith, and C. M. Lieber, Nature(London) 415, 617 (2002).

[2] Y. Wu, R. Fan, and P. Yang, Nano Lett. 2, 83 (2002).

[3] M. T. Bjork, B. J. Ohlosson, T. Sass, A. I. Persson, C. Thelander, M. H. Magnusson, K. Deppert, L. R. Wallenberg, L. Samuelson, Nano Lett. 2, 87 (2002).

[4] R. Solanki, J. Huo, and J. L. Freeouf and B. Miner, Appl. Phys. Lett. 81, 3864 (2002).

[5] B. Lassen and M. Willatzen, Phys. Rev. B 74, 035332 (2006).

[6] Aizhen Zhang, L. C. Lew Yan Voon and M. Willatzen, Phys. Rev. B 73, 045316 (2006).

[7] L. C. Lew Yan Voon and M. Willatzen, J. Appl. Phys. 93, 9997 (2003).

[8] M. T. Bjork, B. J. Ohlosson, C. Thelander, A. I. Persson, K. Deppert, L. R. Wallenberg, L. Samuelson, Appl. Phys. Lett. 81, 4458 (2002).

[9] C. Thelander, T. Martensson, M. T. Bjork, B. J. Ohlosson, M. W. Larsson, L. R. Wallenberg, L. Samuelson, Appl. Phys. Lett. 81, 4458 (2002).

[10] A. Kikuchi, M. Kawai, M. Tada and K. Kishino, Jpn. J. Appl. Phys. 43, L1524 (2004).

[11] T. Sekine, S. Suzuki, H. Kuroe, M. Tada, A. Kikuchi, and K. Kishino, e-J Surf. Sci. Nanotech 4, 227 (2006).

[12] S. Mizuno, Phys. Rev. B 71, 085303 (2005).

[13] S. Mizuno, Jpn. J. Appl. Phys. 47, 3817 (2008).

[14] T. Inui, Y. Tanabe, and Y. Onodera, Group Theory of Crystal Lattice and Its Applications in Physics (Springer-Verlag, Berlin, 1996).

[15] C. J. Bradley and A. P. Cracknell, The Mathematical Theory of Symmetry in Solids, (Clarendon Press, Oxford, 1972).

[16] S. Adachi: GaAs and Related Materials: Bulk Semiconducting and Superlattice Properties (World Scientific, 1994).

[17] N. Nishiguchi, Y. Ando, and M. N. Wybourne, J. Phys. 9, 5751 (1997).

[18] B. A. Auld, in Acoustic Fields and Waves in Solids, Vol. 2 (Robert E. Krieger Publishing Company, Malabar, Florida, 1990).

[19] L. D. Landau and E. M. Lifshits: Theory of Elasticity (Course of Theoretical Physics, Vol. 7, 
Pergamon Press, 1986).

[20] N. Nishiguchi, Physica E 13, 1 (2002).

[21] P. A. Knipp and T. L. Reinecke, Phys. Rev. B 52, 5923(1995).

[22] F. T. Vasko and V. V. Mitin, Phys. Rev. B 52, 1500(1995). 